\title{
Increased Volumes of Subcortical Regions in Patients with Alcohol Dependence
}

\author{
Qingyan Yang ${ }^{1}$, Kebing Yang ${ }^{1}$, Yajuan Niu ${ }^{1}$, Fengmei Fan ${ }^{1}$, Song Chen ${ }^{1}$, Xingguang Luo ${ }^{2}$, Shuping Tan ${ }^{1}$, Zhiren Wang ${ }^{1}$, Jinghui Tong ${ }^{1}$, \\ Fude Yang ${ }^{1}$, Chiang-Shan $\mathrm{R} \mathrm{Li}^{2,3}$, and Yunlong $\operatorname{Tan}^{1, *}$
}

${ }^{1}$ Peking University Huilongguan Clinical Medical School, Beijing Huilongguan Hospital, Beijing, People's Republic of China, China

2Department of Psychiatry, Yale University School of Medicine, New Haven, CT 06519, USA

${ }^{3}$ Department of Neuroscience, Yale University School of Medicine, New Haven, CT 06519, USA

*Corresponding author: Yunlong Tan, PhD, Peking University Huilongguan Clinical Medical School, Beijing Huilongguan Hospital, Beijing, PR China, China, Tel: +86-(10)-83024319; Fax: +86-(10)-62710156; E-mail: yltan21@126.com

Received: 02 Jun, 2020 | Accepted: 18 May, 2021 | Published: 26 May, 2021

Citation: Yang Q, Yang K, Niu Y, Fan F, Chen S, et al. (2021) Increased Volumes of Subcortical Regions in Patients with Alcohol Dependence. J Neurol Neurobiol 7(1): dx.doi.org/10.16966/2379-7150.175

Copyright: (C) 2021 Yang Q, et al. This is an open-access article distributed under the terms of the Creative Commons Attribution License, which permits unrestricted use, distribution, and reproduction in any medium, provided the original author and source are credited.

\section{Abstract}

Objectives: This study aimed to compare the volumes of subcortical regions of Individuals with Alcohol Dependence (IADs) with Health Controls (HCs), and investigate the relationship between the volumes and clinical traits in the IADs.

Methods: In present study, 33 male IADs and 35 gender and age matched HCs were recruited. All of the IADs had stayed abstinent from 14 days to 28 days. The volumes of subcortical regions were measured using MRI 3.0T and analyzed by Free Surfer.

Results: Compared to HCs, IADs had significantly increased in the volumes of left and right thalamus, left and right caudate and left accumbens. The volume of the left thalamus was negatively associated with the age of beginning drinking and illness duration in IADs.

Conclusions: IADs were associated with increased volumes of subcortical structures, which were regions affected by damage to and should be recovery from alcohol. Age of beginning drinking and illness duration can be used as a predictor to the risk of damage of left thalamus.

Keywords: Alcohol dependence; Subcortical region; Volume; Magnetic resonance imaging

\section{Introduction}

Accumulating evidence suggested there were morphometric changes in the brains of patients with alcohol dependence [1], which involved in detrimental cerebral alterations, global and regional cerebral atrophy and white matter lesions [2-4]. Some studies revealed gray matter shrinkage in the bilateral prefrontal cortex, bilateral insula, and bilateral posterior cingulate cortex in the patients with alcohol dependence [5]. Subcortical regions might be under the impact of chronic alcohol use and have relation with domains disrupted by addiction [6]. Most research findings suggested that patients possessing alcohol dependence decreased volume in subcortical regions, such as the hippocampus $[7,8]$, Nucleus Accumbens (NAc) [9], caudate, and putamen [10]. On the contrary, a study found that individuals undergoing long-term abstinence did not manifest a reduced volume in subcortical regions, manifesting the potentiality for recovery in structure at the abstinence period [11]. Moreover, a previous study reported that IADs with obvious brain volume loss at the baseline experienced greater recovery during abstinence [12,13]. Studies also found more rapid brain tissue gain during the first month of abstinence [12].
The morphometric changes in the brains of IADs may be affected by their clinical features. For example, in a study of IADs adolescents, researchers found that the thalamic and putamen volumes of male IADs adolescents were less than those of male non-drinking ones, but those of female IADs adolescents were greater in contrast with female non-drinking ones [14]. Weissman DG, et al. found an earlier age of alcohol use is associated with increased resting-state functional connectivity between prefrontal and NAc [15]. And the duration of abstinence was found be positively correlated with volume in subcortical regions [16].

Given that subcortical structures are an important location in addiction; this study is intended for exploring whether the subcortical structural alterations are affected by excessive alcohol consumption. We also explore whether it will undergo significant recovery within 2-4 weeks of abstinence in IADs. We Hypothesis: 1) There are differences in subcortical structures between IADs and HCs; 2) The subcortical volumes may express a compensated changing after a short-term abstinence from excessive alcohol consumption (increased or decreased); 3) The subcortical regional volumes may be correlated with the clinical features of IADs. 


\section{Materials and Methods}

\section{Participants and consents}

All patients were recruited from the Beijing Huilongguan Hospital between the years 2017 and 2018. Individuals with alcohol dependence in this study were all Han Chinese. Inclusion criteria were as follows: (a) met the criteria for alcohol dependence according to the Diagnostic Statistical Manual of Mental Disorders (DSM-IV) [17]; (b) aged between 18 and 65 years; (c) right-handed; (d) had completed medically assisted withdrawal and had stayed abstinent from 14 days to 28 days, with no clear withdrawal symptoms, and the total score of Clinical Institute Withdrawal Assessment for Alcohol (CIWA) [18] should be less than 3. Exclusion criteria were as follows: (a) the occurrence of other Axis-I mental disorders that met DSMIV criteria, (b) current dependence upon substances apart from alcohol and nicotine (in line with the M.I.N.I.-plus International Neuropsychiatric Interview) [2] and (c) accompanied with severe somatic diseases (eg., unstable hypertension or diabetes, myocardial infarct, liver cirrhosis).

In the study, we recruited 35 ages and education matched male healthy controls (HCs) without major medical or mental illness history from the local community. Each participant should provide written informed consent before their research inclusion. The study obtained the approval of the ethics review board of Beijing Huilongguan Hospital, China.

\section{Clinical assessment}

The clinical assessments were completed as follows: 1) All IADs were diagnosed with alcoholic dependence, in line with the M.I.N.I.plus. 2) Alcohol Use Disorder Identification Test (AUDIT) [19] should be utilized for IADs in assessing the severity of alcoholism. 3) A semistructured questionnaire was used for investigating their lifetime alcohol consumption [20]. From the lifetime drinking history, daily drinks (containing 10 grams of ethanol per standard drink), and age of first drinking, age of onset and duration of alcohol dependence were calculated. 4) All HCs received an MRI scan, and IADs received MRI scan after an acute detoxification.

\section{MRI scan}

The MRI results of the participants could be achieved by using a Siemens 3T Prisma MRI scanner, involving 33 patients and 35 healthy controls. Structural MRI parameters had been obtained including the whole brain possessing sagittal 3D-Magnetization Prepared Rapid Acquisition Gradient Echo (MPRAGE) sequence: Echo Time (TE) = $2.98 \mathrm{~ms}$, Inversion Time (TI) $=1100 \mathrm{~ms}$, Repetition Time $(\mathrm{TR})=2530$ $\mathrm{ms}$, Flip Angle $(\mathrm{FA})=7^{\circ}$, Field Of View $(\mathrm{FOV})=256 \mathrm{~mm} \times 224 \mathrm{~mm}$, matrix size $=256 \times 224$, thickness $/$ gap $=1 / 0 \mathrm{~mm}$.

\section{Structural MRI data analysis}

Subcortical volumes had been acquired utilizing free surfer $[21,22]$ containing volumes of the left and right lateral ventricle, thalamus, caudate, putamen, pallidum, NAc, hippocampus, amygdala and Total Gray Volume (TGV). The TGV worked as a covariate in analysis to explain distinctions amid head sizes. For the sake of quality control, the ENIGMA pipeline (http://enigma.ini.usc.edu/) was obeyed: all Regions of Interest (ROIs) with a volume $>1.5$ or $<1.5$ times the interquartile scope was found and visually examined by eye through superimposing their segmentations on the anatomical images of subjects. Merely ROI data for which segmentation had been determined by visual inspection as accurate were subjected to statistics analysis.

\section{Statistics analysis}

Categorical data were used by chi-square $\left(\chi^{2}\right)$ test. Except gender (all participants are male), analysis of other demographics (age, years of education, smoking status) was performed through independent sample t-tests. Data of consequent variables should be expressed to be average \pm standard deviation. The statistics threshold was set at $\mathrm{p}<0.05$ with the two-tailed t-test. For each volume of all 14 subcortical regions $(7 \times 2$ bilateral ones), group comparisons between HCs and IADs were conducted with univariate linear regression analysis, where all subcortical volumes of ROIs were the dependent variable and the group (IADs or HCs), age, smoking status, education, and TGV were taken as covariates, through a p-value threshold of 0.0035714 (i.e., $0.05 / 14$ ) for Bonferroni correction for diverse comparison.

We identified 5 ROIs which were significantly different between IADs and HCs. To explore the relationship between the 5 different subcortical regions and clinical features using age, education, smoking status and TGV as covariates. With utilization of multiple linear regression analysis, the relation was assessed amid the clinical characters and the abnormal brain regions. This also included variables with reference to the results of the univariate analysis. $\mathrm{P}<0.05$ was considered to be the significant difference. All the statistical analysis procedures should be conducted utilizing SPSS 20.0 (IBM, USA).

\section{Results}

\section{Demographics and clinical features}

Table 1 showed the demographics and clinical features of the participants. There were no significant differences in age $(t=1.257$, $\mathrm{p}=0.213)$ and education $(\mathrm{t}=-0.883, \mathrm{p}=0.381)$ between IADs and HCs, while there were differences between IADs and HCs on smoke $\left(\chi^{2}=11.87, \mathrm{p}=0.001\right)$. In the whole analyses, the variables age, education and smoke were used as covariates.

\section{MRI data: the mancova}

Compared to controls, patients showed significantly larger volumes in the left thalamus, right thalamus, left caudate, right caudate, and left accumbens ( (all p<0.0036 (0.05/14) Table 2 and Figure 1) with age, education, smoke, and total gray volume as covariates.

\section{Multiple linear regression analysis}

As shown in table 3 , the volume of left thalamus in IADs had a negative correlation with the age of beginning drinking ( $\beta=-0.438$, $\mathrm{p}=0.008)$ and illness duration $(\beta=-0.386, \mathrm{p}=0.019)$. No significant correlation was shown between the other aberrant subcortical regions and clinical features in IADs ( $\mathrm{p}>0.05)$.

\section{Discussion}

Damages in functionality of several brain areas makes the neural mechanism of alcoholic dependence very complex. Despite the fact that a tremendous increase has been seen in neurobiological explorations on alcohol dependence $[23,24]$, the neural mechanism of alterations in functional network patterns is still unclear. MRI has provided a noninvasive means of exploring volumetric and morphometric changes correlated with alcohol dependence.

Many studies have reported reductions in the volume of subcortical regions in IADs [14,25-27]. Presenting the potentiality for several structure recovery at the abstinent period [14,25]. Similar to our findings, Sullivan and colleagues revealed larger NAc in sober alcoholics in contrast with recent drinkers [10]. Mohammad Sameti [11] found that there were no differences between long-term abstinent 
Table 1: Participant demographics and clinical characteristics.

\begin{tabular}{|l|c|c|c|}
\hline & IADs (n=33) & HCs (n=35) & t/ $\boldsymbol{\chi}^{\mathbf{2}-\text { value }}$ \\
\hline Age, years & $44.8 \pm 9.8$ & $41.6 \pm 11.5$ & 1.257 \\
\hline Education, years & $11.8 \pm 3.3$ & $12.3 \pm 2.4$ & -0.883 \\
\hline Smoking/non-smokinga* & $26 / 7$ & $13 / 22$ & 11.87 \\
\hline Age of onset, years & $32.5 \pm 10.1$ & N/A & N/A \\
\hline Age of beginning drinking, years & $19.9 \pm 5.5$ & N/A & N/A \\
\hline AUDDIT total score & $21.7 \pm 5.2$ & N/A & N/A \\
\hline Mean daily drinking & N/A & N/A \\
\hline
\end{tabular}

Notes: a: Chi-square $\left(\chi^{2}\right)$ test; data was showed by mean \pm standard deviation values, ${ }^{*} \mathrm{p}<0.05$.

Abbreviations: IADs-Individuals with Alcohol Dependence; HCs-Health Controls; AUDIT-Alcohol Use Disorders Identification Test; N/A, Not Applicable. B: A drink contains 10 grams of pure alcohol.

Table 2: The volumes of subcortical regions between patients with alcoholism and healthy controls.

\begin{tabular}{|c|c|c|c|c|}
\hline Volume of subcortical structure & IADs $(n=33)$ & HCs $(n=35)$ & F-value & p-value \\
\hline Left thalamus & $12333.8 \pm 2896.3$ & $8002.1 \pm 772.9$ & 49.97 & 1.63E-09 \\
\hline Right thalamus & $10431.5 \pm 2039.4$ & $7523.8 \pm 720.5$ & 51.68 & $1.01 \mathrm{E}-09$ \\
\hline Left caudate & $3645.3 \pm 841.5$ & $3423 \pm 409.5$ & 18.72 & $5.60 \mathrm{E}-05$ \\
\hline Right caudate & $3670.9 \pm 856.5$ & $3480.3 \pm 405$ & 9.72 & 0.003 \\
\hline Left putamen & $3694 \pm 1077.3$ & $5096.2 \pm 512.8$ & 8.75 & 0.004 \\
\hline Right putamen & $3901.5 \pm 1118.3$ & $5191.3 \pm 538.5$ & 5.64 & 0.021 \\
\hline Left pallidum & $1707.4 \pm 539.3$ & $2129 \pm 196.9$ & 7.67 & 0.007 \\
\hline Right pallidum & $1791.8 \pm 477.2$ & $2104.3 \pm 215$ & 4.46 & 0.039 \\
\hline Left hippocampus & $4044.5 \pm 557.2$ & $4015.3 \pm 322.8$ & 3.35 & 0.072 \\
\hline Right hippocampus & $3781.7 \pm 619.1$ & $4233.3 \pm 289.4$ & 1.66 & 0.202 \\
\hline Left amygdala & $1563.5 \pm 288.6$ & $1686.3 \pm 169.1$ & 0.29 & 0.595 \\
\hline Right amygdala & $1703.1 \pm 331.4$ & $1779.4 \pm 187.3$ & 0.66 & 0.418 \\
\hline Left accumbens & $579.9 \pm 210.3$ & $435.9 \pm 91.1$ & 19.17 & $4.68 \mathrm{E}-05$ \\
\hline Right accumbens & $558.2 \pm 187.3$ & $505.8 \pm 85$ & 7.534 & 0.008 \\
\hline
\end{tabular}

Note: data was showed by mean \pm standard deviation values; One-way ANOVA, represents Bonferoni corrected significantly $p<0.004$ $(p<0.05 / 14=0.004)$.
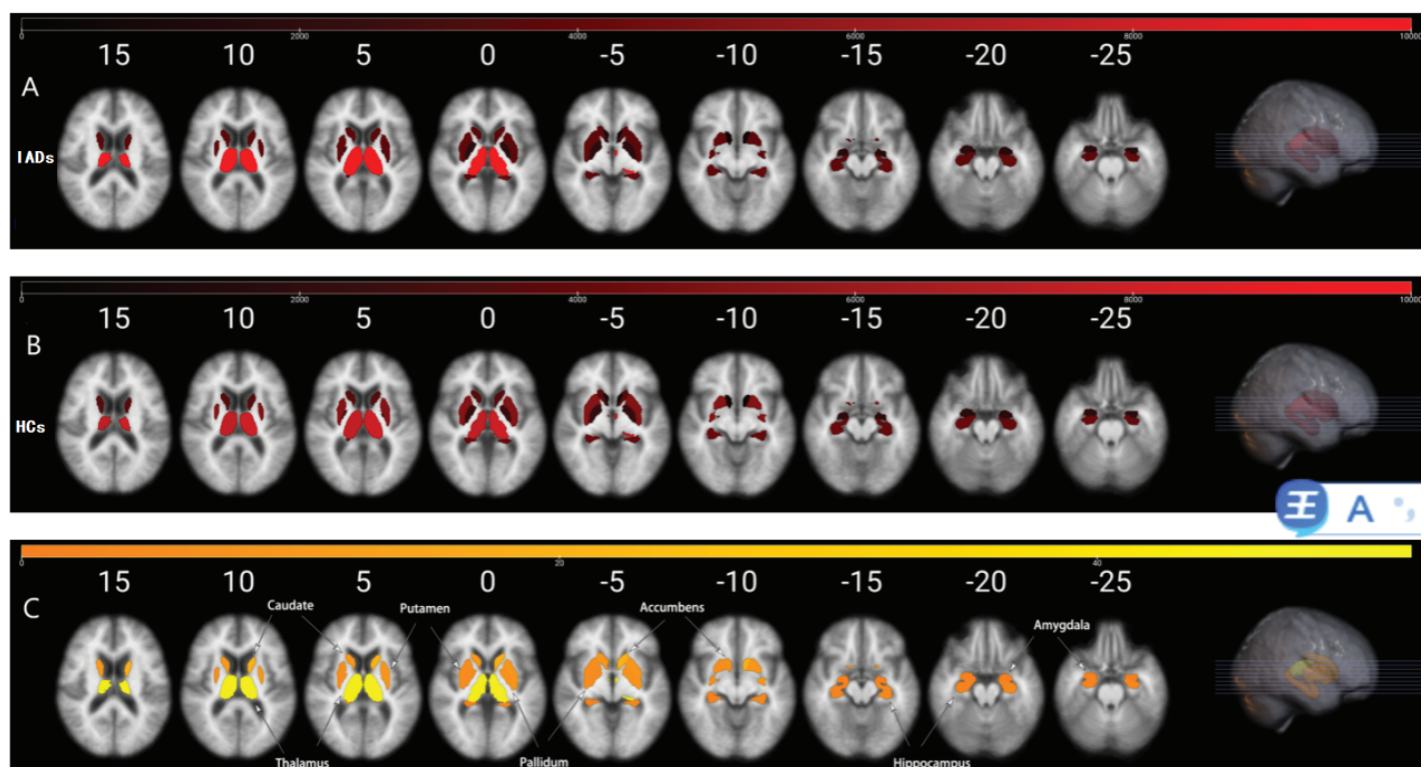

Figure 1: MRI images of patients with alcoholism and healthy controls.

Note: A: Individuals with Alcohol Dependence (IADs); B: Health Controls (HCs); C: there are significant differences in volume of left and right thalamus, left and right caudate, and left accumbens between IADs and HCs, $p<0.004(p<0.05 / 14=0.004)$. 
Table 3: Relationship between clinical characteristics and brain regions of interests.

\begin{tabular}{|l|c|c|c|c|c|}
\hline & \multicolumn{5}{|c|}{ Dependent variables: Left thalamus } \\
\hline Predictors & B & SE & $\beta$ & $p$ & $r^{2}$ \\
\hline $\begin{array}{l}\text { Age of beginning } \\
\text { drinking, years }\end{array}$ & -228.72 & 81.031 & -0.438 & 0.008 & 0.119 \\
\hline $\begin{array}{l}\text { Illness duration, } \\
\text { years }\end{array}$ & -145.73 & 58.528 & -0.386 & 0.019 & 0.246 \\
\hline
\end{tabular}

alcoholics and health [15]. These different brain morphological alterations may indicate the different staging of alcoholism like intoxication and detoxification. For example, increased subcortical brain volumes may reveal abstinent alcoholics recover subcortical brain volumes possessing continuous abstinent status.

Erica found that the uses of multiple substances have interaction with alcohol, can protect subcortical volumes from decrease, and cause the subcortical volumes in at least one region grow [27]. Such research outcomes suggest that the uses of extra substances are likely to protect the subcortical area from alcohol induced damage by inflammation or possibly by acting in a protection way [14]. In our study, there are significant differences in smoke between IADs and HCs, most of IADs smoke, and few HCs smoke. Researches on human being revealed extensive effects of nicotine and smoking on brain functionalities [28]. The imbalance of smoke status in two groups may be the main reason for the results of the study.

General linear regression analysis showed that these two indexes (age of beginning drinking and illness duration) might be associated with the change of the left thalamus. The thalamus is associated with compulsive behaviour, because compulsive self-stimulation can be found in patients possessing thalamus implanted stimulation electrodes [29], serving as a significant relay station from the striatum to the orbitofrontal cortex. Thalamic volume decrease in alcohol dependent and psycho stimulant dependent comorbidities has been seen [30,31]. Other research on an identical group of people found no reduction in thalamic volume [11]. According to this study, the younger age of beginning drinking, the more obvious the compensatory increase after abstinence. Conversely, if the drinking damage continues, the longer the time, the more obvious the brain damage.

Some limitations in this study should be considered. First, most of patients were also cigarette smokers, but only a few controls were smokers in the current study. Researches about human being revealed nicotine and smoking is associated with changes in subcortical structures [32]. However, smoke as one of covariates was considered in the analyses. Second, this study was observational instead of experimental. There is uncertainty about the difference amid IADs and HCs samples due to alcohol, not several unmeasured cohort distinctions amid samples. Third, IADs in our study were abstinent for 2-4 weeks. This research is therefore reticent regarding subcortical volume effects of both alcohol and recovery in abstinence. Such effects are likely to appear in the first days and weeks of abstinence.

Ultimately, our results were different from those of most related studies, because we did not actually measure atrophy, which has been commonly studied in the past. To our knowledge, this is one of but few neuroimaging studies that reported increased subcortical volumes in alcohol dependent individuals. Given that IADs in this study had been abstinent from alcohol for 14 days to 28 days, our results may reflect both the effects of alcohol and individual recovery. Since IADs participated in the study after at least 2 weeks of treatment, the volume of subcortical changes resulting from the influence of medication cannot be ruled out. In future studies, MR spectroscopy studies could be used, which was able to make measurement of subcortical neuronal markers and metabolites reflecting inflammations. Inflammations and shrinkage may be deduced based on this kind of study in a more direct manner.

\section{Conclusion}

Heavy drinking could cause temporary or continuous damage to brain. In our study IADs were associated with increased volumes of subcortical structures, which were regions affected by damage to and should be recovery from alcohol. Age of beginning drinking and illness duration might be a predictor to the risk of damage of left thalamus.

\section{Acknowledgements}

All authors declared that they have no conflicts of interest to this work. We declare that we do not have any commercial or associative interest that represents a conflict of interest in connection with the work submitted.

\section{Compliance with Ethical Standards}

\section{Conflict of interest}

The authors declare that they have no conflict of interest.

\section{Funding}

This work was supported by the Beijing Municipal Administration of Hospitals Clinical medicine Development of special funding support (XMLX201834) and the National Institutes of Health (AA021449).

\section{References}

1. Bühler M, Mann K (2011) Alcohol and the human brain: a systematic review of different neuroimaging methods. Alcohol Clin Exp Res 35: 1771-1793.

2. van Vliet IM, de Beurs E (2007) The MINI-International Neuropsychiatric Interview. A brief structured diagnostic psychiatric interview for DSM-IV en ICD-10 psychiatric disorders. Tijdschr Psychiatr 49: 393-397.

3. Zahr NM (2014) Structural and microstructral imaging of the brain in alcohol use disorders. Handb Clin Neurol 125: 275-290.

4. Xiao P, Dai Z, Zhong J, Zhu Y, Shi H, et al. (2015) Regional gray matter deficits in alcohol dependence: A meta-analysis of voxel-based morphometry studies. Drug Alcohol Depend 153: 22-28.

5. Sutherland GT, Sheedy D, Kril JJ (2014) Neuropathology of alcoholism. Handb Clin Neurol 125: 603-615.

6. Cardenas VA, Durazzo TC, Gazdzinski S, Mon A, Studholme C, et al. (2011) Brain morphology at entry into treatment for alcohol dependence is related to relapse propensity. Biol Psychiatry 15: 561-567.

7. Silvers JM, Tokunaga S, Berry RB, White AM, Matthews DB (2003) Impairments in spatial learning and memory: ethanol, allopregnanolone, and the hippocampus. Brain Res Brain Res Rev 43: $275-284$.

8. Lee J, Im SJ, Lee SG, Stadlin A, Son JW, et al. (2016) Volume of hippocampal subfields in patients with alcohol dependence. Psychiatry Res Neuroimaging 258: 16-22.

9. Makris N, Oscar-Berman M, Jaffin SK, Hodge SM, Kennedy DN, et al. (2008) Decreased volume of the brain reward system in alcoholism. Biol Psychiatry 64: 192-202. 
10. Sullivan EV, Deshmukh A, De Rosa E, Rosenbloom MJ, Pfefferbaum A (2004) Striatal and forebrain nuclei volumes: contribution to motor function and working memory deficits in alcoholism. Biol Psychiatry 57: 768-776.

11. Sameti M, Smith S, Patenaude B, Fein G (2011) Subcortical volumes in long-term abstinent alcoholics: associations with psychiatric comorbidity. Alcohol Clin Exp Res 35: 1067-1080.

12. Gazdzinski S, Durazzo TC, Meyerhoff DJ (2005) Temporal dynamics and determinants of whole brain tissue volume changes during recovery from alcohol dependence. Drug Alcohol Depend 78: 263-273.

13. Eijk JV, Demirakca T, Frischknecht U, Hermann D, Mann K, et al. (2013) Rapid partial regeneration of brain volume during the first 14 days of abstinence from alcohol. Alcohol Clin Exp Res 37: 67-74.

14. Fein G, Fein D (2013) Subcortical volumes are reduced in short-term and long-term abstinent alcoholics but not those with a comorbid stimulant disorder. Neuroimage Clin 3: 47-53.

15. Weissman DG, Schriber RA, Fassbender C, Atherton O, Krafft C, et al. (2015) Earlier adolescent substance use onset predicts stronger connectivity between reward and cognitive control brain networks. Dev Cogn Neurosci 16: 121-129.

16. Korponay C, Kosson DS, Decety J, Kiehl KA, Koenigs M (2017) Brain Volume Correlates with Duration of Abstinence from Substance Abuse in a Region-Specific and Substance-Specific Manner. Biol Psychiatry Cogn Neurosci Neuroimaging 2: 626-635.

17. American Psychiatric Association (2000) Diagnostic and Statistical Manual of Mental Disorders, (4 $\left.{ }^{\text {th }} \mathrm{Ed}\right)$, Text Revision (DSM-IV-TR). American Psychiatric Association, Washington, DC, USA: 915.

18. Sullivan JT, Sykora K, Schneiderman J, Naranjo CA, Sellers EM (1989) Assessment of alcohol withdrawal: the revised clinical institute withdrawal assessment for alcohol scale (CIWA-Ar). Br J Addict 84: 1353-1357.

19. Saunders JB, Aasland OG, Babor TF, de la Fuente JR, Grant M (1993) Development of the Alcohol Use Disorders Identification Test (AUDIT): WHO Collaborative Project on Early Detection of Persons with Harmful Alcohol Consumption-II. Addiction 88: 791-804.

20. Sobell LC, Brown J, Leo GI, Sobell MB (1996) The reliability of the Alcohol Timeline Followback when administered by telephone and by computer. Drug Alcohol Depend 42: 49-54.
21. Fischl B (2012) FreeSurfer. Neuroimage 62: 774-781.

22. Fischl B, Salat DH, Busa E, Albert M, Dieterich M, et al. (2002) Whole brain segmentation: automated labeling of neuroanatomical structures in the human brain. Neuron 33: 341-355.

23. Everitt BJ, Belin D, Economidou D, Pelloux Y, Dalley JW, et al. (2008) Review. Neural mechanisms underlying the vulnerability to develop compulsive drug-seeking habits and addiction. Philos Trans R Soc Lond B Biol Sci 363: 3125-3135.

24. Koob GF, Volkow ND (2010) Neurocircuitry of addiction. Neuropsychopharmacology 35: 217-238.

25. Benegal V, Antony G, Venkatasubramanian G, Jayakumar PN (2007) Gray matter volume abnormalities and externalizing symptoms in subjects at high risk for alcohol dependence. Addict Biol 12: 122132

26. Wang GY, Demirakca T, Eijk JV, Frischknecht U, Ruf M, et al. (2016) Longitudinal Mapping of Gyral and Sulcal Patterns of Cortical Thickness and Brain Volume Regain during Early Alcohol Abstinence. Eur Addict Res 22: 80-89.

27. Grodin EN, Momenan R (2017) Decreased subcortical volumes in alcohol dependent individuals: effect of polysubstance use disorder. Addict Biol 22: 1426-1437.

28. Gallinat J, Meisenzahl E, Jacobsen LK, Kalus P, Bierbrauer J, et al. (2006) Smoking and structural brain deficits: a volumetric MR investigation. Eur J Neurosci 24: 1744-1750.

29. Portenoy RK, Jarden JO, Sidtis JJ, Lipton RB, Foley KM, et al. (1986) Compulsive thalamic self-stimulation: a case with metabolic, electrophysiologic and behavioral correlates. Pain 3: 277-290.

30. Mon A, Durazzo TC, Abe C, Gazdzinski S, Pennington D, et al. (2014) Structural brain differences in alcohol-dependent individuals with and without comorbid substance dependence. Drug Alcohol Depend 144: 170-177.

31. Fein G, Greenstein D, Cardenas VA, Cuzen NL, Fouche JP, et al. (2013) Cortical and subcortical volumes in adolescents with alcohol dependence but without substance or psychiatric comorbidities. Psychiatry Res 214: 1-8.

32. Das D, Cherbuin N, Anstey KJ, Sachdev PS, Easteal S (2012) Lifetime cigarette smoking is associated with striatal volume measures. Addict Biol 17: 817-825. 